\title{
Patterns of Blood Flow in Episcleral Vessels Studied by Low-Dose Fluorescein Videoangiography.
}

\author{
PAUL A. R. MEYER \\ Cambridge
}

\section{Summary}

The blood supply of the ocular anterior segment arises from a saggittal arterial ring composed of the long posterior ciliary arteries, the muscular and anterior ciliary arteries and perforating scleral arteries. This ring supplies coronal arterial circles within and outside the globe.

Low dose anterior segment fluorescein videoangiography demonstrates arterial and venous flow, recording its characteristics and direction.

Videoangiograms were performed at low and high magnification in 15 normal subjects. Episcleral arteries and veins were distinguishable by the presence or absence of pulsatile flow and by their fluorescence intensity. Arteries usually perfused earlier than veins, and with higher flow velocity.

Twenty-five of $\mathbf{4 0}$ arteries flowed away from scleral perforations close to the limbus. All 8 veins drained away from the limbus.

The communication between two arteries may be demonstrated by a static, pulsating column of non-fluoresceinated blood. This sign arose at a point of scleral perforartion, at the junction between muscular and anterior ciliary arteries and in the episcleral arterior circle. It supports the concept of arterial shunting, both in the saggittal plane and in the superficial coronal circle.

Perforating scleral arteries lay anterior to the episcleral arterial circle in superior angiograms and posterior to it in inferior studies.

Vessels that had been characterised by videoangiography were identified in stereo colour photographs of angiographic fields. Arteries were best distinguished from veins by their high tortuosity and thick walls.

Using these photographic characteristics, the distribution of arteries and veins over the rectus muscles was surveyed in 13 subjects. The incidence of arteries is lowest over the lateral rectus muscle. Veins were concentrated in the vertical meridian and were absent over lateral rectus in 8 subjects.

Five intra-operative videoangiograms are reported. Retraction of conjunctiva facilitated imaging of the episcleral vessels and demonstrated arterial communication through a shared capillary bed.

Although the vessels of the ocular anterior segment comprise the most accessible circulation in the body, controversy surrounds the patterns of blood flow within them.

The brilliant dissections of Leber ${ }^{1}$ form the foundation for our current understanding of this circulation. His findings have since been embellished by indian ink injections ${ }^{2}$ and dissections of vascular castings . $^{3,4,5,6}$

On the surface of the eye the muscular arteries, which arise from the ophthalmic artery $^{7}$, run forward as the anterior ciliary 
arteries $^{1}$ and meet in an anterior episcleral arterial circle (eac.). ${ }^{6.8}$ This feeds the anterior conjunctival and episcleral capillary beds $^{8}$. Within the globe the medial and lateral long posterior ciliary arteries, which also arise from the ophthalmic artery ${ }^{7}$, supply the "major circle of the iris". 1,5,6 This is thought to distribute blood to the ciliary body, iris and anterior choroid 5 .

The intra-ocular arterial circles may be more variable and fragmented than Leber believed $^{9}$, and methacrylate castings of primate anterior segment vessels ${ }^{6}$ indicate that it has two components: one at the root of the iris and one within the ciliary muscle itself.

The two anterior arterial circles communi-

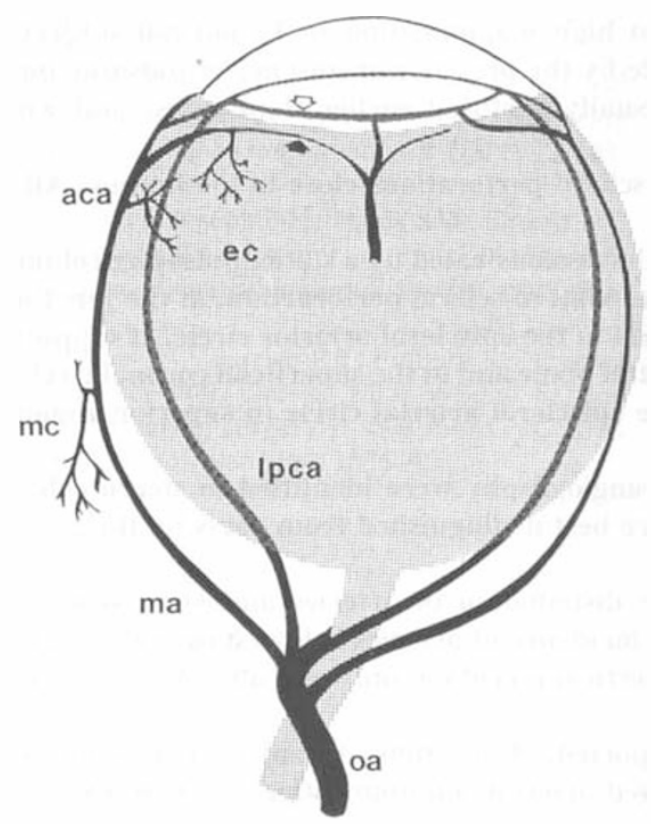

Fig. 1. Arterial communications in the anterior segment of the globe.

oa - ophthalmic artery

ma - artery of rectus muscle

aca-anterior ciliary arteries (joined by episcleral arterial circle)

lpca - long posterior ciliary artery

ec-episcleral capillaries

mc - muscular capillaries

$\checkmark$ - intraocular arterial circle (major circle of iris)

- extraocular arterial circle (anterior episcleral arterial circle)

note: $m a+$ aca + scleral perforator + lpca form saggittal arterial ring. cate by perforating branches of the anterior ciliary arteries. ${ }^{5,6}$ The potential therefore exists for an elaborate arterial shunt system, in which two coronal arterial circles, one within and one outside the globe, are supplied and joined by a saggittal arterial ring (Fig. 1).

In order to clarify the routing of blood through this system, many authors have examined the direction of flow in photographic fluorescein angiograms of anterior ciliary vessels. Bron and Easty ${ }^{10}$, Amalric, Rebiere and Jourdes ${ }^{11}$, Ikegami, Talusan and Schwartz ${ }^{13}$ and Watson and Bovey ${ }^{14}$ have all collected angiograms of large episcleral vessels that penetrated the sclera close to the limbus, and in which blood flow appeared to be centrifugal.

However, the interpretation of their results is complicated by the presence of "emissary veins" that cross the supra-choroidal space and perforate the sclera, draining the iris and ciliary body circulations into the episcleral venous system. ${ }^{2}$ Ikegami, and Talusan and Schwartz asserted that perforating centrifugal scleral vessels were anterior ciliary arteries, but Almaric et al. and Bron and Easty considered them to be "emissary veins".

Photographic anterior segment fluorescein angiography has been subject to two important constraints: fluorescein extravasation may interfere with documentation of the capillary and venous phases, and the slow recycling rate of flash units restricts observations on flow characteristics or direction. Both these problems are overcome by low dose anterior segment fluorescein videoangiogaphy.

Intravascular fluorescein binds ionically to circulating albumin ${ }^{15,16}$ and, by restricting the dose to avoid saturating this system, fluorescein leakage may be considerably reduced. ${ }^{8}$

Since a video camera captures 25 frames per second, each of which is composed of two fields, the direction of flow with velocity of up to $400 \mathrm{~mm} / \mathrm{sec}$. can theoretically be determined in an $8 \mathrm{~mm}$ diameter field (assuming uniform illumination, a sharp fluorescein front and no camera sensitivity threshold). Flow characteristics such as pulsatility are also demonstrated. 
Videoangiography does pose technical problems. Continual illumination of the eye demands a lower intensity of exciting radiation, there is consequently less fluorescence, and a very sensitive video camera is required. As a result, intensified silicon target (ISIT) cameras have been used for posterior segment fluorescein videoangiography. ${ }^{17,18}$

During anterior segment fluorescein angiograms, low fluorescence is compounded by the need to restrict the dose of fluorophore. A further complication is the very high fluorescence that accrues late in the angiogram as a result of fluorescein leakage from conjunctival and episcleral capillaries. This requires the camera to tolerate a range of luminance far greater than an ISIT camera can accomodate.

These requirements are satisfied by "second generation" image intensifiers. The image is formed on a photocathode, from which electrons are accelerated and focussed onto a micro-channel-plate. This acts as a two-dimensional array of photomultipliers, which further accelerate the electons to excite an output phosphor. The recreated image can be recorded by a sensitive monochrome video camera.

The present study uses this technology to establish patterns of arterial perfusion and venous drainage in the anterior segment of the human eye. This is essential for a clear understanding of ocular physiology and for the rational design of surgical procedures.

\section{SUBJECTS AND METHODS}

\section{Episcleral Fluorescein Videoagiography Without Intervention}

\section{Subjects}

Fifteen normal subjects participated: eight females and seven males. Their ages ranged from 23 to 62 years. All gave informed consent.

The right eye was studied in six subjects and the left eye in nine. Each participant underwent two angiograms, separated by an interval of more than twenty-four hours: the first included the inferior, nasal and temporal episclera at "x10" magnification $(13 \mathrm{~mm} / \mathrm{ver}$ tical screen). From this field an area of interest including the limbus, and at least one major anterior ciliary vessel, was selected for the second angiogram at " $x 16$ " magnification $(8 \mathrm{~mm} /$ vertical screen $)$. The subject looked ahead during the first angiogram: subsequent angiograms at " $\mathrm{x} 16$ " were performed at extremes of horizontal or vertical gaze.

In three subjects 3 additional angiograms were performed at " $\mathrm{x} 16$ " magnification, to complete a survey of the anterior globe.

The anterior segment vasculature of each eye was also recorded on colour reversal film (Echtochrome, ASA 200): a survey of the whole anterior segment circulation was made at " $\mathrm{x} 10$ " and stereo pairs were taken of every angiographic field.

\section{Videoangiography Recording Technique}

Angiograms were performed on a Ziess clinical slit lamp. Excitation of flourescence was by a 25 watt tungsten source with a 450$490 \mathrm{~nm}$ band-pass interference filter, and a long pass barrier filter (T50:515 nm) was placed in the imaging light path.

For video recording the image was reflected by a $50 / 50$ beam splitter onto the photo-cathode of a second generation image intensifier (Mullards XX1500), fibre-optically coupled to a Nuvicon monochrome camera (Visual Contacts). The photocathode potential of the image intensifier was controlled manually and the camera was modified to give linear electronic gain.

Images were stored on low-band U-matic videotape. For analysis they were transfered with a time-base onto 1 inch videotape, slowed to one fifth real time and re-recorded in U-matic format.

The still reproductions in this paper were prepared by grabbing paused frames from the original tapes into a digital frame store (Supernova).

\section{Procedure}

Each angiogram was preceded by a $30 \mathrm{sec}$. video sequence of the selected area, allowing an appropriate image intensifier gain setting to be selected. This was chosen to be the lowest gain that gave a negative image of vessels against background scleral auto- or pseudo fluorescence. The camera settings (electronic gain and black level) were not changed between subjects.

The position of any pseudofluorescence 
was determined from the control sequences, and artefacts arising from light reflexes were recorded by displacing the light source.

Sodium fluorescein $(1.5 \mathrm{mg}$ per kilogram body weight) was then injected rapidly into an anti-cubital fossa vain. The recording continued throughout the circulation time and the arterial, capillary and venous phases of the angiogram. It was terminated when fluorescein leakage had negated all positive vascular images.

\section{Interpretation of Angiograms}

The low power videoagiograms were examined to determine gross patterns of episcleral and conjunctival perfusion, and to select areas suitable for further investigation. The directions of flow within large vessels were noted wherever possible and compared with those undertaken at extremes of gaze.

All quantitative work was performed upon angiograms that had been recorded at high magnification.

\section{Large Vessels}

All vessels that were well focussed, and imaged with adequate contrast, were drawn on a flow diagram, and where possible their depth was found from stereo colour photographs.

\section{Perfusion Characteristics}

The angiographic sequence was timed from the first appearance of intravascular fluorescein.

The following criteria were recorded for at least six vessels in each angiographic field:

(1) The moment of first perfusion with fluorescein.

(2) The presence or absence of pulsatile (discontinuous) flow.

(3) Fluorescence intensity (analogue scale 03 , where $0=$ no positive image; $3=$ saturation of grey scale).

(4) Flow velocity and direction (whenever possible).

\section{Flow Velocity}

Flow velocity was estimated only in stretches of vessel that demonstrated a sharp fluorescein front, were more than $4 \mathrm{~mm}$ long and lacked any run-off. Observations upon arteries were confined to systole.

The position of the fluorescein column was recorded on a paused frame and the number of fields that elapsed before the column reached a second, predetermined position were recorded. The sequence was then continued until the vessel had perfused fully before the length between the two points were measured with dividers. Corrections were made for magnification on the monitor.

\section{Microcirculation}

At present videoangiography does not achieve the same resolution as photographic imaging and the interpretation of capillary detail was often restricted. Where possible (5 angiograms), the origin and drainage of capillaries were recorded, but no attempt was made to estimate systolic flow velocity. In the remaining angiograms, study of the microcirculation was limited to the recording of fields of perfusion.

\section{Intra-Operative Videoangiography of Episclera}

Angiograms of exposed muscular and episcleral vessels were performed in five adult subjects during cataract or detachment surgery. The superior rectus was examined in three patients and studies were also performed over the inferior and lateral recti.

Conjunctiva over the region to be examined was retracted using the minimum possible dissection and cautery. Muscles were relaxed, and no attempt was made to measure or correct intra-ocular pressure.

The angiography proceedure was similar to that described above. Off-axis illumination of the operating field was provided by a 50 watt quartz-halogen light source, transmitted by a fibreoptic light guide fitted with a condenser. A 50/50 beam splitter, mounted on a Zeiss operating microscope, directed the image through the long-pass barrier filter onto the photocathode of the image-intensified video camera. The fluorescein dose was $2.0 \mathrm{mg} / \mathrm{kg}$.

\section{Identification of Vessels in Colour Photographs}

Where possible, the large radial vessels in which videoangiographic flow characteristics 
had been recorded were identified in the stereo colour photographs of angiographic fields. Their photographic appearances were scored as follows:

(1) tortuosity (the least angle between tangents to the circumference of a bend was measured (Fig. 3); radius of curvature was not considered. $<90^{\circ}=$ high; $>90^{\circ}=$ low).

(2) Wall thickness (1-3, where visible blood column $=1$; vessel elevated when viewed stereoscopically $=3$ ).

(3) Streaming of blood $(+/-)$.

Having established photographic characteristics arteries and veins, the vessels seen in $360^{\circ}$ photographic surveys of 13 normal eyes were identified and drawn. The incidence of episcleral arteries and veins at each rectus muscle insertion was recorded.

\section{Results}

\section{Gross Perfusion Patterns}

The whole globe did not fluoresce simultaneously: The episclera was perfused in a number of discrete fields which filled at intervals of up to 13.5 seconds. Although pigment may interfere with the iris angiogram, perfusion of the iris was seen to precede that of the episclera in 4 of the 15 studies.

The anterior perfusion fields were usually centred upon single or grouped perforating episcleral arteries that flowed away from the limbus and towards the rectus muscles. When grouped, vessels at the centre of a field fluoresced simultaneously. Adjacent fields were joined by the episcleral arterial circle (eac.), which supplied the intervening episclera (Fig.5).

Venous blood also drained towards the rectus muscles (Fig.2), however venous and arterial fields did not always coincide.

Iris perfusion was similarily sectorial.

\section{Discrimination Between Episcleral Arteries and Veins.}

Angiographic Appearance (Figs. 2,4,5)

Large, radial episcleral vessels were allocated to two categories, based upon their flow characteristics:

(I) Vessels that filled early in a perfusion field were highly fluorescent $(>=2$ on analogue scale) and had pulsatile flow.

These were considered to be arteries. Sys- tolic flow velocity was very variable $(1.6-$ $100 \mathrm{~mm} / \mathrm{sec}$.), but all high velocity vessels were in this group.

(II) Major vessels that filled after the arterial flow phase in the field concerned were usually less fluorescent ( $=2$ on analogue scale), and sometimes distinguishable only by the loss of their vascular silhouette. Their flow was not pulsatile.

Vessels with these characteristics were considered to be veins. They filled gradually and with a diffuse fluorescein front, hence the moment of their first perfusion, their flow direction and velocity were difficult to ascertain. When this was possible, flow velocity $(<4.3 \mathrm{~mm} / \mathrm{sec}$. $)$ was usually lower than within adjacent arteries.

Photographic Appearance (Fig. 3)

It was possible to record all the chosen angiographic and photographic criteria in 50 large radial vessels from 18 high power angiograms. These had been performed in 14 subjects, two of whom contributed 3 studies.

Thirty five vessels with the angiographic characteristics of arteries were found to have thick walls and high tortuousity. One, though thick-walled, was not tortuous.

Seven vessels with the angiographic characteristics of veins had low tortuosity and thin walls. One vein, which emerged from the sclera and received other episcleral veins, had low tortuousity but thick walls.

Six vessels (from 2 subjects) had high tortuousity and thick walls, but filled late in the angiogram. In one subject, both vessels with these characteristics demonstrated pulsation.

On the basis of these findings, tortuousity and wall thickness were chosen as photographic discriminants between arteries and veins:

arteries: tortuousity high; wall thickness $>=$ 2. veins: tortuousity low; wall thickness $<=$ 2.

In addition, arteries branched little and never showed laminar flow, whereas veins were highly branched and 4 out of 8 revealed streaming of blood.

Photographic Surveys (Fig. 6)

All the radial episcleral vessels in each quadrant of 13 eyes (13 subjects) were classified as 

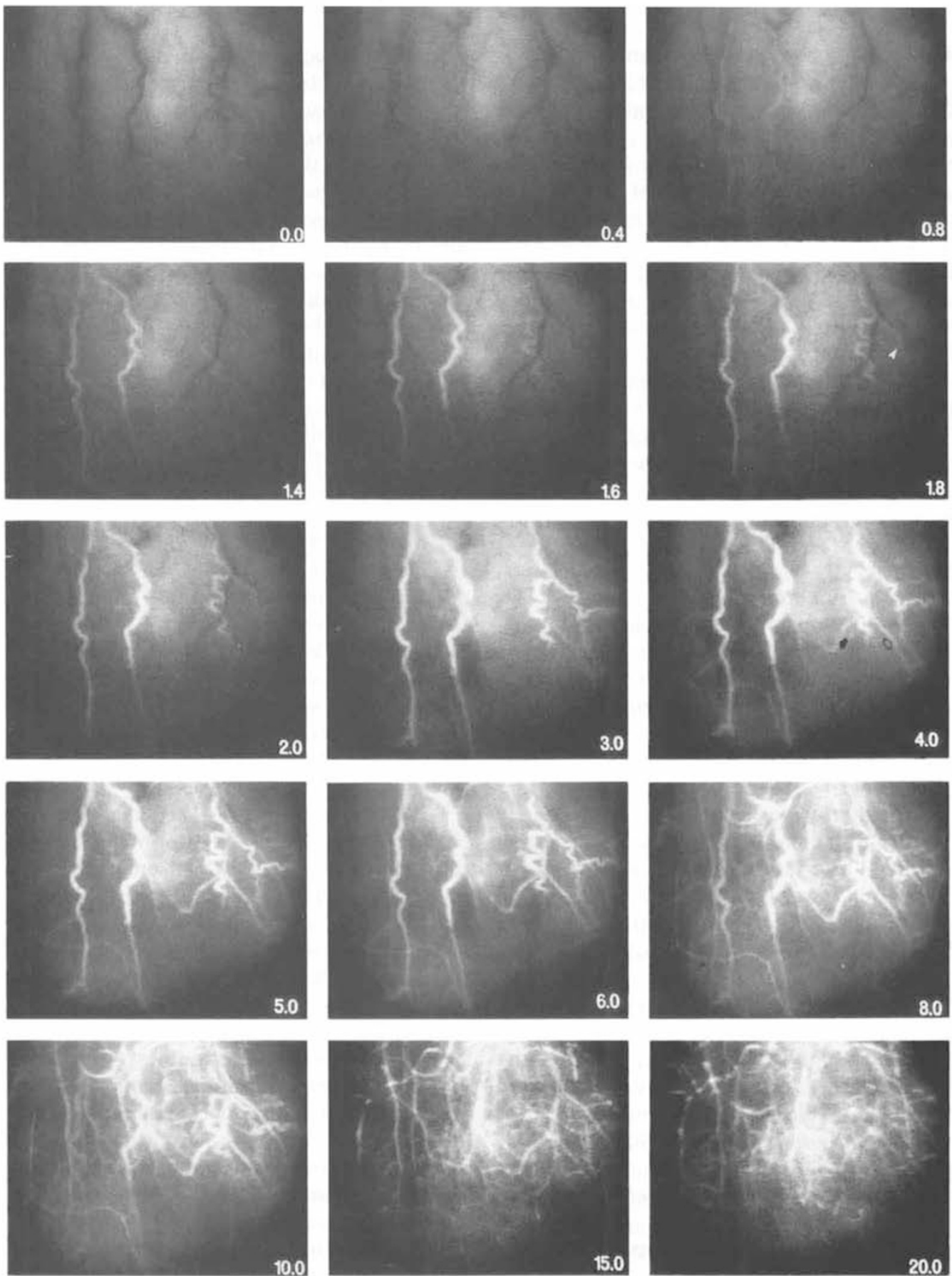

Fig. 2. Superior angiogram

(imagnification: $8 \mathrm{~mm} /$ vertical field. timing: seconds after first flush)

Top row: centrifugal filling of two adjacent anterior ciliary arteries, comprising a perfusion field.

Second row: Single centrifugal anterior ciliary artery supplies adjacent perfusion field. This gives rise to episcleral arterial circle ( ) which is fully perfused by 4 secs.

Third row: centrifugal filling of the episcleral venous system. A superficial episcleral vein ( () is joined by an emissary vein (\$).

Fourth row: the late venous phase.

This fades (20.0 secs), leaving beading of the fluorescein column in conjunctival vessels that perfuse intermittently. 
arteries or veins according to the above photographic criteria. In vessels that were not identifiable by major criteria (tortuousity and wall thickness) alone, the number of their branches, and the characteristics of the vessels that flowed from or into them, were considered in addition. The mean incidence of arteries and veins over each muscle is illustrated in Fig. 6. The incidence of arteries over the lateral rectus is lower than in the other quadrants. Veins were concentrated in the vertical meridian, and eight out of 13 subjects had no temporal vein.

\section{Arteries}

Direction of Blood Flow

Forty radial arteries and 8 radial veins from 16 angiograms were adequately imaged for flow direction to be documented.

Twenty-five episcleral arteries flowed away from the limbus: 15 carried blood towards the limbus. Arteries flowing in opposing directions were found in nine out of 21 angiographic fields (Fig. 7).

All veins drained away from the limbus (Fig. 2).

\section{Scleral Perforators and the Episcleral Arterial Circle}

Twenty-four arteries perforated the anterior sclera in 16 angiograms. Two flowed back as anterior ciliary arteries, making no contribution to the eac; one fed the eac, but did not give rise to an anterior ciliary artery: the remainder supplied both circulations.

The perforating arteries were classified according to their relationship to the eac: anterior (7), beneath (11), posterior (6).

No single angiogram demonstrated arteries emerging from the sclera both anterior and posterior to the eac. Perforations were situated anterior to the eac. in both superior angiograms and posterior in both inferior studies. Their disposition was variable in the horizontal meridian.

\section{Arterial Shunts}

Communications between arteries in the anterior segment fell into two categories: Indirect, in which two arteries shared a capillary bed.
Direct, in which vessels joined end to side, or end to end.

\section{Indirect.}

The superimposition of conjuctival and episcleral capillary beds made indirect communication difficult to establish in most angiograms. However it was confirmed in episcleral capillaries at the rectus muscle insertion in one intra-operative angiogram, in which the conjunctiva had been retracted (Fig. 7).

\section{Direct (Fig. 5)}

The eac. was imaged in all 15 low power angiograms and ten of these demonstrated direct arterial communications within it.

Arteries from different sources usually joined at the site of a shared capillary bed. However, when merging vessels each supplied separate run-offs, the intervening column of blood remained static, pulsating, and non-fluoresceinated. Fluorescence spread gradually, presumably as a result of diffusion of the dye.

The circumstances giving rise to this sign were not often found. However, the eac. was demonstrated in this way in 3 angiograms; communication between episcleral arteries flowing away from the limbus and muscular arteries in 3 angiograms; and communication "between the eac. and a deep circulation (presumed to be the intra-ocular arterial circle) in one angiogram.

\section{Capillaries (Fig. 4)}

In four of the 5 angiograms in which the con-

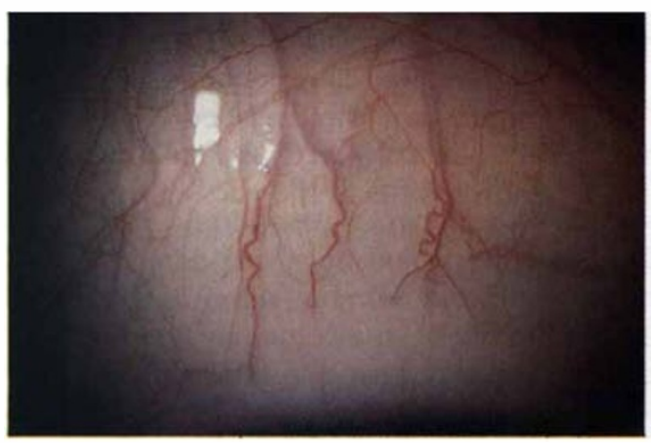

Fig. 3. Photograph of angiographic field shown in Fig. 2. 

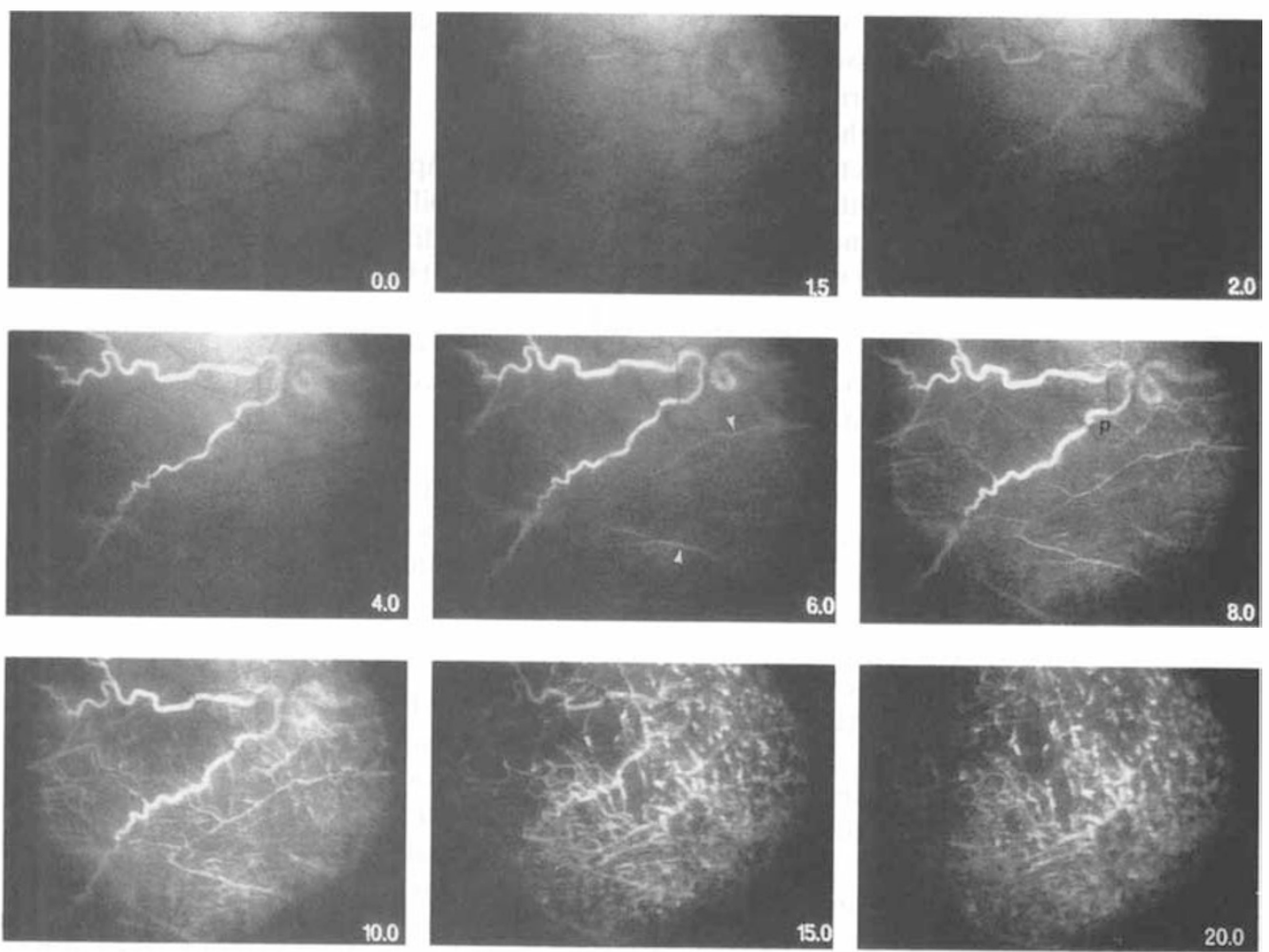

Fig. 4. Left temporal videoangiogram

( $8 \mathrm{~mm} /$ vertical field; seconds after first flush)

Top row: filling of bifid anterior ciliary artery away from the limbus.

Second row: filling of the episcleral arterial circle begins at 4 sec. and is complete at 8 sec. It supplies anterior conjunctiva and episclera.

The posterior tarsal conjunctiva is supplied by a loop composed of two posterior tarsal arteries ( ). Conjunctival capillaries derived from these and from the episcleral arterial circle communicate.

Pulsation was seen between an anterior ciliary artery and muscular artery ( $p)$.

junctival and episcleral capillary beds were adequately imaged, episcleral capillaries arose from the eac and from branches of radial anterior ciliary arteries. In the remaining angiogram, episcleral detail was masked by the conjunctival capillary bed.

The anterior conjunctival capillaries filled from anterior roots of the eac, or equivalent vessels arising directly from anterior ciliary arteries.

\section{Veins}

Eight radial episcleral veins could be fully characterised angiographically. They flowed back towards a rectus muscle, accumulating episcleral branches. Photographs showed the episcleral contributions to arise as follows (Fig. 8):

1. Circumferential limbal venules that lie anterior to the eac, draining radial conjunctival capillaries and the limbal arcades.

2. Veins that drain the episcleral capillary net.

3. 4/8 of these veins received a deep contribution.

Veins that emerged from the sclera were distinguishable in stereo colour photographs into two groups: fine, thin-walled vessels that gradually surfaced as they flowed away from the limbus and large, thick-walled vessels that passed through distinct scleral foramina. Although only one of the latter 

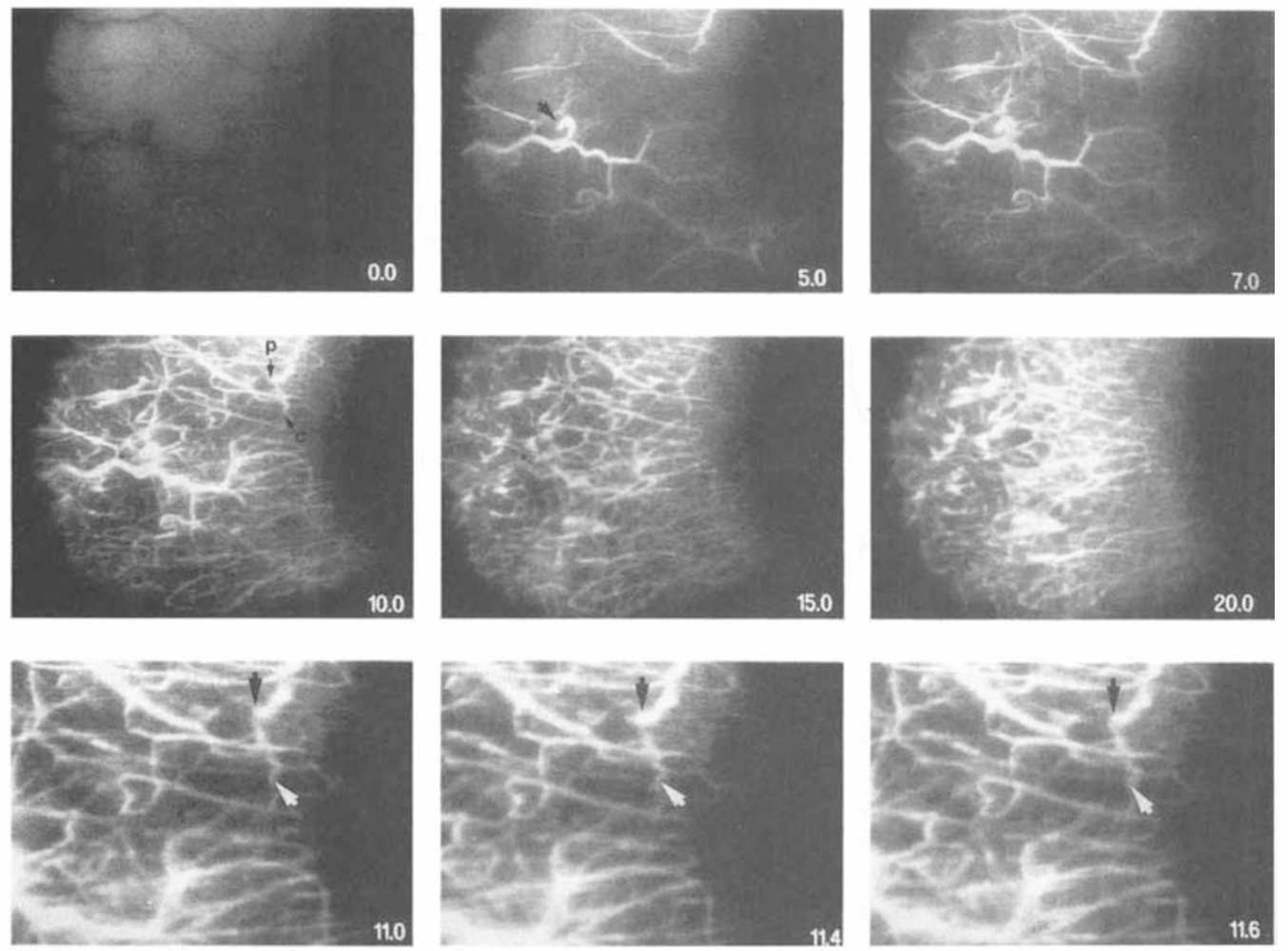

Fig. 5. Right temporial angiogram

(8mm/vertical field; seconds after first flush)

Filling emanates from a perforator (arrow). Note the pulsating dye fronts boardering non-perfused segments of perforating scleral artery (p) and episcleral arterial circle (c); (10sec.).

Bottom row: (4mm/vertical field) illustrates a single cardiac cycle (arrows remain unchanged).

was seen in the high-power studies, review of the low-power angiograms revealed similar vessels in a further two subjects.

Venous anastomoses were frequently apparent on colour photography. Since emissary veins presumably transmit intraocular pressure modulations, pulsation of venous blood may have been expected where they merged with episcleral veins, or within superficial inter-venous shunts. Although no venous communication was revealed in this way in fluorescein videoangiograms of intact eyes, this sign was found in an intra-operative study over the inferior rectus muscle (v.i.).

Intra-Operative Angiography.

The distributions of the major vessels over superior rectus in the three subjects are illus- trated in Figure 9. Arterial flow was predominantly away from the limbus in two subjects. and towards the limbus in one. All three angiograms showed veins flowing onto the superior rectus muscle, but one field had an additional venous sytem flowing towards the limbus (the only veins to do so in the whole study).

In the angiogram over the inferior rectus muscle, the major arterial flow was away from the limbus. A single small artery left the muscle to supply episclera close to its insertion, and one perfusion field received blood from both afferent and efferent arterioles. In this subject venous drainage of the whole inferior episclera converged upon a single vessel that ran back over the muscle. Pulsation was clearly visible within this venous system. 


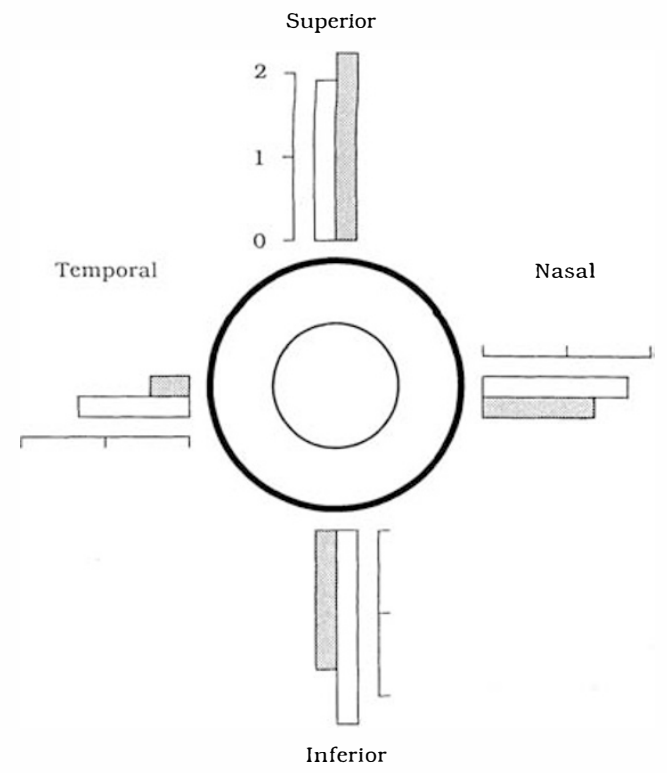

Fig. 6. Mean incidence of arteries (clear) and veins (shaded) over each rectus muscle.

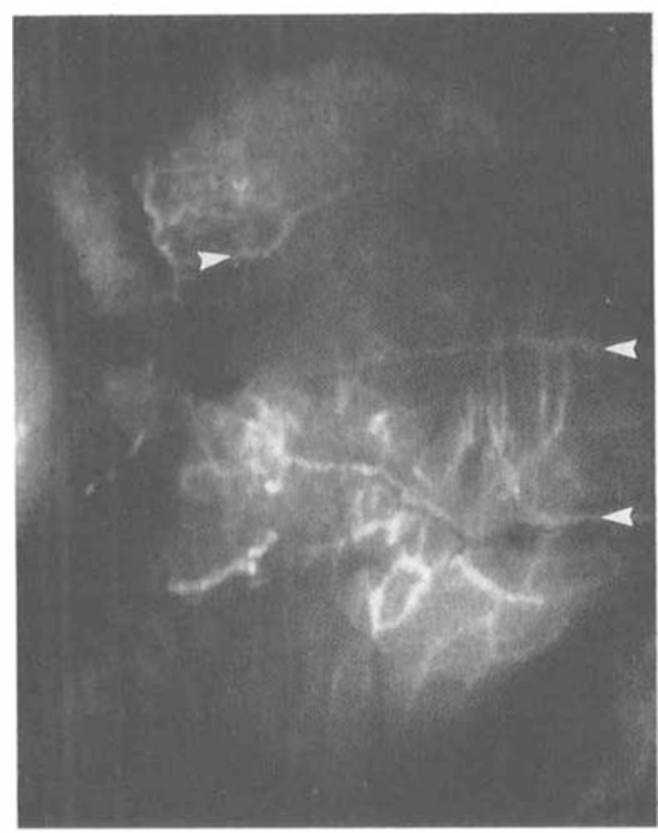

(a)
An intraoperative angiogram over the lateral rectus muscle is illustrated in Figure 7.

\section{Discussion}

Fluorescein videoangiography is a powerful research technique with which to study the anterior segment circulations. Arteries and veins may now be distinguished by their flow characteristics, flow direction can be recorded, and flow velocity estimated. The results have resolved many paradoxes arising from previous work, and should rationalise the planning of anterior segment surgery.

\section{The Arterial Rings of the Anterior Segment}

The anatomy of arterial communications in the anterior segment offers the potential for a network of shunts, in which the coronal arterial circles (one within and one outside the globe) are fed and joined by a saggittal arterial ring (vs).

The superficial (episcleral) arterial circle

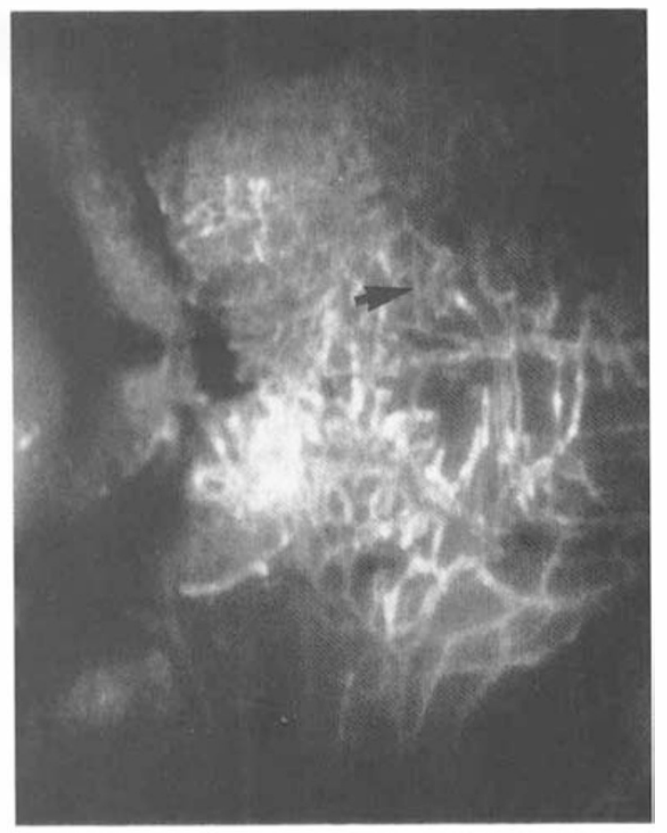

(b)

Fig. 7. Intra-operative angiogram over lateral rectus muscle. (Conjunctiva has been retracted to expose only episcleral and muscular vessels).

a. Arterial phase: Arrows show flow direction in anterior ciliary arteries.

b. Capillary phase: Arrow indicates communication between anterior ciliary arteries through the episcleral capillary bed. 
has been confirmed. Anterior ciliary arteries commonly flow away from the limbus over all quandrants. Since only two long posterior ciliary arteries exist, both in the horizontal meridian, $1,5,6$ blood must be delivered to the superior and inferior anterior ciliary arteries by an intra-ocular arterial circle.

In some angiograms direct communication between arteries was indicated during the flow phrase of the angiogram by columns of static, pulsating, non-fluorescent blood. This sign was discovered in a perforating anterior ciliary artery, and between anterior ciliary arteries and muscular arteries close to the rectus muscle insertion: it indicates two sites at which the muscular and long posterior ciliary contributions to the saggittal arterial ring do indeed meet.

This sign requires each limb of a shunt to have a separate run-off, and one of these must lie within the angiographic field. These circumstances arose in only four out of 15 angiograms. Although it indicates the existance of saggittal arterial shunting, it does not establish its incidence.

Flow Dynamics in the Saggittal Arterial Ring The direction of flow at any point within the saggittal arterial ring is presumably determined by resistance within its component arteries and by the impedence of the circulations that it supplies. These influences may be expected to vary under physiological and pathological conditions, or following surgery.

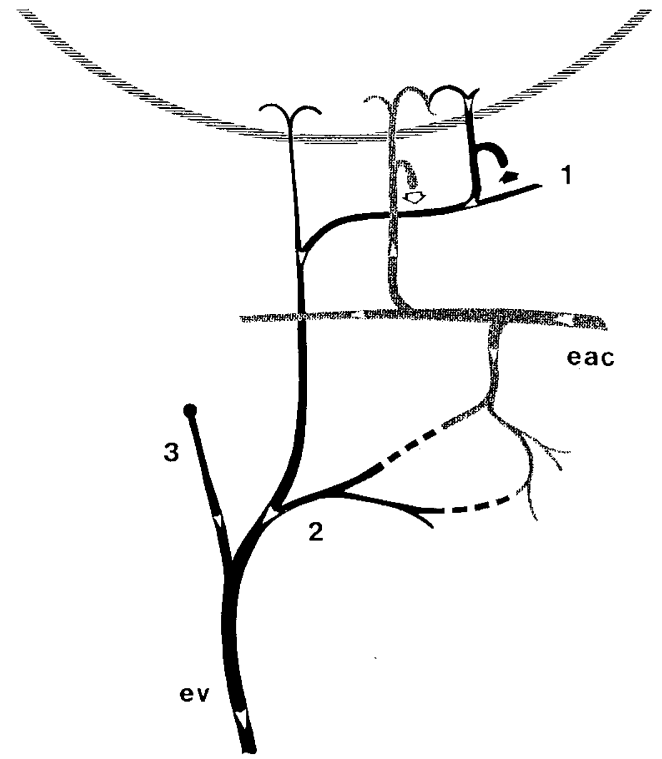

Fig. 8. Episcleral venous drainage.

Arteries shaded, veins black. Arrows indicate origins of conjunctival arteries and veins.

eac $=$ episcleral arterial circle.

ev $=$ episcleral vein.

1. circumferential limbal veins (limbal venous circle).

2. episcleral veins.

3. perforating veins (presumed to be emissary veins and veins draining the deep limbal microcirculation).
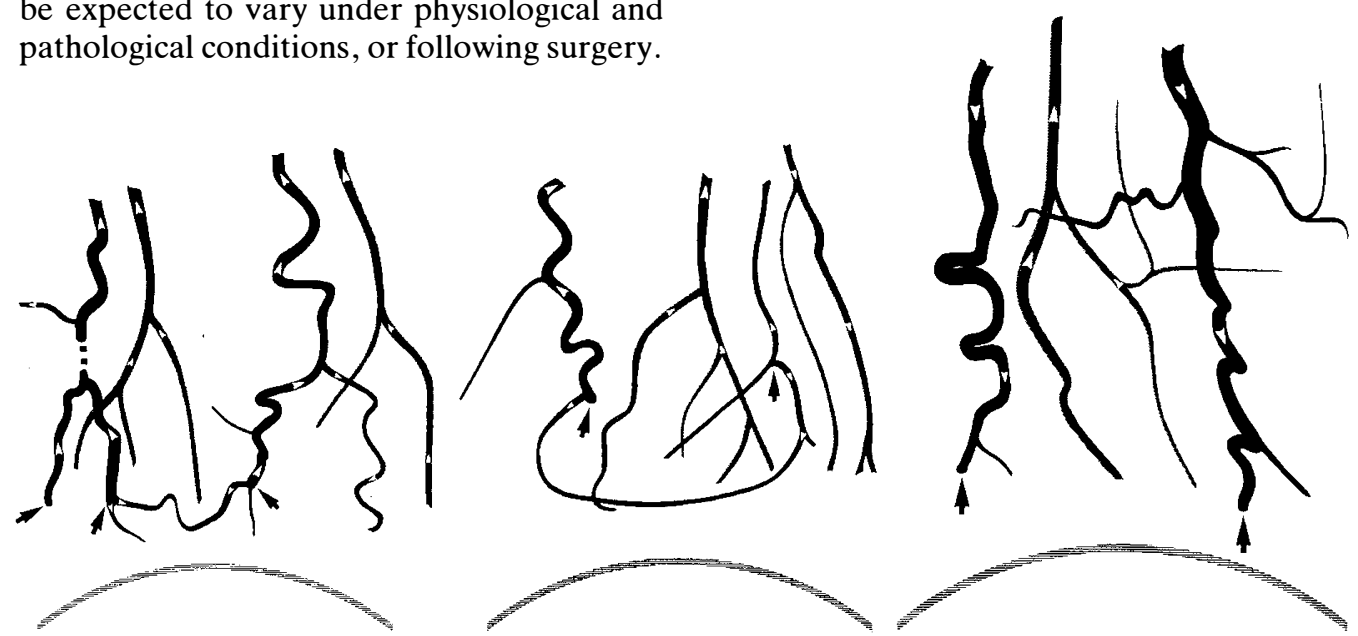

Fig. 9. Intra-operative angiograms over superior rectus muscles.

(3 subjects)

Arteries are black, veins shaded. Arrows indicate directions of flow. 
In the angiograms upon which this study is based, most anterior ciliary arteries carried blood away from the limbus, independent of the direction of gaze. Therefore normal subjects derive the main blood supply to their anterior sclera and conjuctiva from within the globe.

The perfusion delays between adjacent episcleral fields presumably reflect the different routes taken by blood within the globe, and confirms the proposal by Singh Hayreh and $S \operatorname{cott}{ }^{9}$ that flow velocity in the intra-ocular arterial circle may be low.

The technique used for flow velocity estimation required the indentification of a point on the advancing fluorescein front in two successive positions on the globe. The diffuseness of this front (particularly in veins), uneven illumination (due to curvature of the globe) and auto- or pseudo-fluorescence all combine to make this technique unreliable for absolute measurements, and more valuable for assessing variations in velocity within a single angiographic field.

Since the anterior ciliary arteries form the apex of an arterial ring, the large range of estimated systolic flow velocity within them is not surprising.

Four out of five intra-operative angiograms also demonstrated anterior ciliary arteries flowing away from the limbus. They either met the muscular vessels to supply episcleral capillaries close to the rectus muscle insertion; or delivered blood into the rectus muscles themselves.

Intra-operative studies over the superior rectus muscles illustrated the variability of flow dynamics at a single antomical site in different subjects. However, further interpretation of these angiograms should be reserved, since the intra-ocular pressure was presumably low, the rectus muscles were relaxed and the conjunctival vessing from the episcleral arterial circle had been cut.

\section{The Venous Drainage of the Anterior Segment}

Venous drainage of the ciliary apparatus and iris has been presumed to be through the emissary veins or vortex veins ${ }^{2}$. This study confirmed the presence of the emissary veins which emerge from the sclera and accumu- late conjunctival and episcleral branches, before passing back over the rectus muscles. This means that both arteries and veins emerge from foramina in the anterior sclera, and all carry blood in the same direction: away from the limbus. However, they are distinguishable by their flow characteristics, enabling morphological differences to be deduced.

Communication between superficial veins in different fields was demonstrated photographically, and the failure of videoangiography to illustrate this probably reflects their numerous tributaries, their lower fluorescence intensity and the diffuse fluorescein front that is found in the venous phase.

In colour photographic surveys, episcleral veins were found to be concentrated in the vertical meridian: particularly over the superior rectus muscle.

\section{Conclusions for the Surgeon}

The anterior segment of the eye has evolved a most elaborate matrix of arterial communications, perhaps in response to a requirement for constant perfusion pressure to the ciliary apparatus. Despite this shunt system, squint or detatchment surgery may sometimes give rise to anterior segment ischaemia.

During detachment surgery, the placement of a plomb or band may occlude a long posterior ciliary artery, breaking the saggittal arterial ring. Additional section of a muscular artery will give rise to anterior segment ischaemia if coronal shunting was inadequate or had been interfered with.

Why transection of muscular arteries alone should cause anterior segment ischaemia is unclear, since this does not disturb the intraocular arterial supply to the iris and ciliary body (the long posterior ciliary arteries and internal coronal arterial circle). It remains possible that the superficial limbs of the saggittal arterial ring are dominant in some subjects.

However, this study has confirmed that emissary veins are a significant route for drainage of the anterior segment, and they leave the anterior episclera over the rectus muscles. There is evidence that the anterior segment ischaemia that may follow rectus 
muscle surgery is the result of venous occlusion (paper in preparation).

Singh Hayreh and Scott ${ }^{9}$ found that sector iris perfusion defects followed section of the vertical, but not the horizontal, recti. They attributed this to anterior ciliary artery occlusion in sectors of the globe that received an inadequate long posterior ciliary artery supply. The discovery that anterior ciliary arteries carry blood away from the limbus in both the vertical and horizontal meridians requires reappraisal of this explanation. Veins are concentrated in the vertical meridian, and venous occlusion may have accounted for the effects of vertical muscle surgery in their patients.

When the ophthalmic surgeon cuts or cauterises vessels he diverts ocular blood flow. The arterial communications of the anterior segment appear to vary in incidence and calibre. When re-routing blood through them, it may be wise to follow the following guidelines:

(1) never interfere simultaneously with both the deep and superficial limbs of either saggittal arterial ring.

(2) avoid disrupting both the saggittal and the coronal arterial circles.

There is little evidence of effective venous shunting in the anterior segment. Furthermore, the distribution of anterior segment veins is concentrated in the vertical meridian. Therefore,

(3) if surgery is to involve one or both vertical rectus muscles, the adequacy of the remaining veins should be ensured.

It is hoped that the characterisation of these vessels in this paper will facilitate this.

This study has demonstrated communication between the intra-ocular, and extraocular veins that drain the anterior segment: the pressure within episcleral, conjuctival and emissary veins must therefore be equal. Intra-ocular veins remain patent only if their intra-luminal pressure exceeds aqueous pressure. Therefore the pressure within the episcleral venous system is equal to intraocular pressure, less the gradient imposed by the impedence of perforating veins. Although this impedence may vary considerably, it must be low in the many subjects in whom these vessels are large. Episcleral, venous and intra-ocular pressure must be virtually equal in these patients.

The destination of aqueous draining from surgical fistulae is still a matter of controversy, however episcleral veins have been proposed. ${ }^{19}$ If this is correct, the impedence of trans-scleral veins may determine the success of these procedures.

Inflammatory disorders of the anterior sclera and cornea are associated with arterial closure ${ }^{14,20}$ and Meyer and Watson ${ }^{8}$ proposed that interruption of flow in the episcleral arterial circle may be responsible. However, if the anterior conjunctiva and episclera are normally supplied from within the globe, and arterial shunting can occur in the saggittal plane, closure of the intra-ocular arterial circle or scleral perforators must also be considered.

Anterior segment fluorescein videoangiography has proved to be a powerful method, yielding dynamic as well as morphological data. It has shed new light upon the vagiaries of normal anterior segment blood flow, and it is perhaps a matter of time before it becomes a clinical investigative technique.

I would like to thank all the subjects who participated in this study, and $\mathrm{Mr} \mathrm{A.T.} \mathrm{Moore} \mathrm{and} \mathrm{Mr.} \mathrm{A.}$ Elliott who performed the dissections for the intraoperative angiograms.

I am most grateful to $\mathrm{Mr} \mathrm{A}$. Quested and $\mathrm{Mr} \mathrm{A}$. Lacey (Medical Illustration Department, M.E.H., City Road) who prepared the animations for the presentation upon which this paper is based.

This study was generously supported by the Iris Fund for the Prevention of Blindness.

Paul Meyer is in receipt of a Welcome Research Fellowship.

\section{References}

${ }^{1}$ Leber T: Die Cirkulations- und Ernahrungsverhaltnisse des Auges. (Graefe-Saemisch Handbuch der Gesamten Augenheilkunde.) Leipzig: Engelmann, 1903, 2: ch 1.

2 Kiss F: Der Blutkreislauf der Auges. Ophthalmologica 1943, 106: 225-50

${ }^{3}$ Ashton N: Anatomical study of Schlemm's canal and aqueous veins by means of Neoprene casts. Br. J. Ophthalmol. 1951, 35: 291-303.

${ }^{4}$ Ashton N: Anatomical study of Schelmm's canal and aqueous veins by means of Neoprene casts. Part II: aqueous veins. $B r$. J. Ophthalmol. 1952, 36: 265-7.

${ }^{5}$ Ashton N and Smith R: Anatomical study of Schlemm's canal and aqueous veins by means 
of Neoprene casts. Part III: arterial relations of Schlemm's canal. Br. J. Ophthtalmol. 1953, 37: $577-86$.

${ }^{6}$ Morrison JC and Van Buskirk EM: Anterior collateral circulation in the primate eye. Ophthalmology 1983, 90: 707-15.

${ }^{7}$ Sing Hayreh S and Dass R: The ophthalmic artery II. Intra-orbital course. Br. J. ophthalmol. 1962, 46: 165-85.

8 Meyer PAR and Watson PG: Low dose Fluorescein angiography of the conjuctiva and episclera. Br. J. Ophthalmol. 1987, 71: 2-10.

${ }^{9}$ Singh Hayreh S and Scott WE: Fluorescein iris angiography. II Disturbances in iris circulation following strabismus operation on the various recti. Arch. Ophthalmol. 1978, 96: 1390-400.

${ }^{10}$ Bron $\mathrm{AJ}$ and Easty DL: Fluorescein angiography of the globe and anterior segment. Trans Ophthalmol. Soc. UK. 1970, 90: 33967.

${ }^{11}$ Amalric P, Rebiere P, Jourdes JC: Nouvelles indications de l'angiographie fluoresceinique du segment anterieur de l'oeil. Ann. Oculist. 1971, 204: 455-68.

${ }^{12}$ Ikegami M: Fluorescein angiography of the anterior ocular. segment. Part 1. Hemodynamics in the anterior ciliary vessels. Acta Soc. Ophthalmol. Jpn. 1974, 78: 39-53.
${ }_{13}$ Talusan ED and Schwartz B: Fluorescein angiography: demonstration of flow pattern in anterior ciliary arteries. Arch. Ophthalmol. 1981, 99: 1074-80.

${ }^{14}$ Watson PG and Bovey E: Anterior segment fluorescein angiography in the diagnosis of scleral inflammation. Ophthalmology 1985, 92: $1-11$.

${ }^{15}$ Brubaker RF, Penniston JT, Grotte DA, Nagataki S: Measurement of fluorescein binding in human plasma using fluorescence polarization Arch. Ophthalmol. 1982, 100: 625-30.

${ }^{16}$ Palestine AG and Brubaker RF: Plasma binding of fluorescein in normal subjects and in diabetic patients. Arch. Ophthalmol. 1982, 100: 1160-1.

17 Haining WM: Video funduscopy and fluoroscopy. Br. J. Ophthalmol. 1981, 65: 702-6.

${ }^{18}$ Favilla I, Barry WR, Turner IJ: Video and digital fluorescein angiography. Aust. N. Zealand J. Ophthalmol. 1986, 14: 229-34.

19 Benedikt O: Zur Wirkungsweise der Trabekulektomie. Klin. Monatsbl. Augenheilkd. 1975, 167: 679-85.

20 Watson PG and Booth-Mason S: Fluorescein angiography in the differential diagnosis of sclerokeratitis.Br. J Ophthalmol. 1987, 71: $145-51$. 